\title{
Functional neurological disorder as a modern paradigm of hysteria: A clinical case
}

\author{
Diana Galletta*, Francesca Di Costanzo and Fausta Micanti \\ Unit of psychiatry and psicology - University hospital "Federico II" Naples, University of Naples Federico II, Via Pansini 5, 80122 Naples, Italy
}

\begin{abstract}
Background: In accordance with the latest edition of the DSM-5, Conversion Disorder is recognized as Functional Neurological Disorder, characterized by the presence of one or more muscle or sensory organs-related symptoms not explained by a clear neurological disease.

Sampling and methods: The authors present a case report of Conversion Disorder or Functional Neurological Disorder. They report the case of a woman who experienced motor paralysis, dysarthria and falls on the floor with loss of consciousness not explained by a neurological disorder. It was important the collaboration between Neurologists, Psychiatrists and Clinical Psychologists in order to obtain the patient's personality and psychopathological profile for a categorial and dimensional diagnosis. The subject received a psychological assessment using the following tools: Rorschach test, MMPI-2, Progressive Raven Matrices.
\end{abstract}

Results: The patient shows typical symptoms of the Functional Neurological Disorder in absence of organic related lesion.

Conclusions: This nosological entity requires a complex diagnostic process, thanks to the integration of different professionals and their scientific contributions, and demands an accurate differential diagnosis. The Functional Neurological Disorder could be considered the modern frontier of the secular hysteria spectrum.

Further studies would be realised to repeat these results and obtain a deeper psych diagnostic assessment.

\section{Introduction}

The Conversion Disorder or Functional Neurological Disorder, as defined in the latest edition of the Diagnostic and Statistic Manual of Mental Disorders (DSM-5) [1], is characterized by the presence of one or more muscle or sensory organs-related symptoms [2] not explained by a clear neurological disease [3].

The current nosographic classification recognizes more the relationship between somatic symptoms and psychopathology, shifting the focus away from the plausible medical hypothesis about symptoms to that which is experienced in association with the somatic symptomatology [4].

One of the most important risks with functional neurological symptoms is to not give the diagnosis of Functional Neurological Disorder in the presence of symptoms compatible with other mental disorders or with neurophysiological and neuropathological conditions of neurological diseases $[5,6]$. The diseases to be considered are: multiple sclerosis, myasthenia gravis, periodic paralysis, mioglobinuric myopathy, polymyositis, Guillain Barrè syndrome, Progressive Supranuclear Palsy and Huntington's chorea.

However, today the differential diagnosis process opens a debate about the use of terms "psychogenic" or "functional" to describe the symptoms related to the Functional Neurological Disorder $[7,8]$. These definitions make reference to an evoked causal relationship between psychopatological factors and symptoms on the one hand and the attempt to go beyond etiological hypothesis and mind-body dualism [9].

\section{Case presentation}

The patient, a 63-year-old female, belongs to a large family. At 12 years old, she was released into her uncle's care. She had three children, one of them lost after three months of pregnancy.

The woman asked for a medical consultation because of the presence of fatigue and loss of strength in the upper and lower limbs and in the trunk.

She was referred by Neurologists to the psychodiagnostic clinic of the Neuroscience Department of University Hospital "Federico II", Naples, Italy, for a psychiatric assessment in order to obtain a personality profile. After clinical interviews, we administered a battery of psychodiagnostic tests.

From 2009 to 2014, the patient experienced these signs: dizziness with left-sided hyposthenia, headache followed by a sudden loss of postural tone leading to a loss of consciousness, fallen to the ground, deviation of the mouth toward the left side, speech impediment, rigidity associated with right arm palsy and homolateral hand tremors.

Correspondence to: Diana Galletta, Unit of psychiatry and psicology University hospital "Federico II" Naples, University of Naples Federico II, Via Pansini 5, 80122 Naples, Italy, E-mail: diana.galletta@unina.it

Key words: conversion disorder, functional neurological disorder, psychodiagnostics, differential diagnosis

Received: March 13, 2018; Accepted: April 10, 2018; Published: April 13, 2018 


\section{Methods}

The patient received a psychological assessment after a magnetic resonance. The following tools were used: Rorschach test, MMPI-2, Progressive Raven Matrices.

Rorschach test is a psychological test used to analyse personality features and emotional functioning but also for identifying thought disorders. It is made up of 10 tables.

The Minnesota Multiphasic Personality Inventory (MMPI) was first published by the University of Minnesota Press in 1943, then replaced by a modified version, the MMPI-2, in 1989.

It consists of 567 items related to 7 Validity Scales, 10 Basic Clinical Scales, 15 Content Scales and 15 Supplementary Scales.

Progressive Raven Matrices is a test used for measuring abstract reasoning. It contains 60 multiple choice questions.

\section{Results}

The magnetic resonance reports: "The ventricular liquoral system is neither enlarged nor deformed. Encephalon morphology is regular; in the bihemispheric brain white matter there are a lot of vascular gliosis areolas into subcortical and paraventricular position.

Diffusion Tensor Imaging does not show lesions in acute or subacute phase".

\section{Psychodiagnostic evaluation}

Rorschach Test presents a good and mature Ego energy in association with a low and immature emotional, affective and pulsional level, that seems to show the Ego inhibition and control on impulses and needs of instant gratification; emotional tension and generalised anxiety are the consequential effects. The introversive inner life, characterized by the absent exterior expression of emotions, shows a close personality avoiding social relations; the patient's clinical story seems to crystallize these mechanisms. The pulsional activity index is adequate as opposed to control functions.

The patient is extremely rigid and controlled, with structurated rationalistic defense mechanisms that could lead to somatization and acting in.

At Minnesota Multiphasic Personality Inventory -2 the patient refers weakness, nervousness and emotional tension and she uses to develop physical symptoms in a typical stress response. The patient appears passive, dipendent, whiny and searching for care.

Insight skills are poor. Frequently, she expresses depressive mood, anxious restlessness and regressive behaviors.

Somatic symptoms and hypochondriac concerns are a defense against psychotic manifestations. She could show lack of self confidence and feelings of worthlessness, especially in stress situations.

Through the Progressive Raven Matrices evaluation, it is possible to recognize a normal intellectual functioning (Q.I. 95). The adequate completion pattern ability underlines the presence of global knowledge and good analogue skills. Deductive reasoning and abstract thought are sufficient.

\section{Discussion}

Literature reports that the coveted combination between mental disorder and organic pathologies already defined during Charcot times $[10,11]$, find an area of knowledge separated from traditional medicine, thanks to the functional brain lesion concept, that recognized the neurotic dimension related to somatic symptoms; it resolved hysteria enigma [12].

The clinical case observed, presenting the absence of organic signals, urging neurologists to requires other psychodiagnostic deepenings that recognize the functional nature of symptomatology and of the psychopathological profile.

We suppose hysteria conversion disorder guides mental processes, so it needs an accurate exploration of personality profile and of life events in order to obtain a clear psychopatological assessment; it is intended to promote the covet multidisciplinaire alliance between Medicine and Clinical Psychology.

Latest meta-analysis indicate non-Invasive Brain Stimulation efficacy in functional weakness and paralysis [13].

\section{References}

1. Diagnostic and statistical manual of mental disorders (2013) 5th edn. Washington, American Psychiatric Association.

2. Carson AJ, Brown R, David AS, Duncan R, Edwards MJ, et al. (2012) Functional (conversion) neurological symptoms: research since the millennium. $J$ Neurol Neurosurg Psychiatry 83: 842-850. [Crossref]

3. Johnson JG, Cohen P, Kasen S, Brook JS (2006) Dissociative disorders among adults in the community, impaired functioning, and axis I and II comorbidity. J Psychiatr Res 40: 131-140. [Crossref]

4. Boeckle M, Liegl G, Jank R, Pieh C (2016) Neural correlates of conversion disorder: overview and meta-analysis of neuroimaging studies on motor conversion disorder. BMC Psychiatry 16: 195

5. Hales Robert E, Yudofsky Stuart C, Weiss Roberts L (2015) Manuale di Psichiatria American Psychiatric Publishing, Milano: Edra.

6. Steel JC, Richardson JC, Olszewsk J (1964) Progressive supranuclear palsy, a heterogeneous degeneration involving the brain stem, basal ganglia and cerebellum with vertical gaze and pseudobulbar palsy, nuchal dystonia and dementia. Arch Neurol 10: 333-359. [Crossref]

7. Dallocchio C, Marangi A, Tinazzi M (2015) Functional or psychogenic movement disorders: an endless enigmatic tale. Front Neurol 6: 37. [Crossref]

8. Hallett M (2016) Functional (psychogenic) movement disorders - Clinical presentations Parkinsonism Relat Disord 22: 149-152. [Crossref]

9. Janet P (1907) The Major Symptoms of Hysteria. London/New York, Macmillan.

10. Charcot JM (1889) Lectures on the diseases of the nervous system. London, The New Sydenham Society.

11. Freud S (1905) Fragments of an analysis of a case of hysteria. London, Standard Edition.

12. Recalcati M (2016) La clinica psicoanalitica: struttura e soggetto. Milano, Raffaello Cortina Editore.

13. Schönfeldt-Lecuona C, Lefaucheur JP, Lepping P, Liepert J, Connemann BJ, et al (2016) Non-invasive brain stimulation in conversion (functional) weakness and paralysis: A systematic review and future perspectives. Front Neurosci 10: 140. [Crossref]

Copyright: (C2018 Galletta D. This is an open-access article distributed under the terms of the Creative Commons Attribution License, which permits unrestricted use, distribution, and reproduction in any medium, provided the original author and source are credited. 